\title{
Políticas públicas de nutrição para o controle da obesidade infantil
}

\author{
Policies on nutrition for controlling childhood obesity
}

Caio Eduardo G. Reis', Ivana Aragão L. Vasconcelos ${ }^{2}$, Juliana Farias de N. Barros ${ }^{3}$

\section{RESUMO}

Objetivo: Analisar criticamente as políticas públicas de nutrição brasileiras no controle da obesidade infantil.

Fontes de dados: Buscaram-se artigos, ensaios, resenhas, resoluções e legislações nas bases SciELO, Lilacs, PubMed, Biblioteca Virtual em Saúde, Sistema de Legislação em Saúde e Legislação em Vigilância Sanitária, que abordavam políticas públicas de nutrição brasileiras no controle da obesidade infantil, publicados entre 1990 e 2010 . Foram utilizadas as palavras-chaves: "obesidade”, "sobrepeso”, "criança”, “escolar(es)”, "políticas públicas”, "política de saúde”, “política de nutrição", "cantina escolar”, "alimentação escolar”, "propaganda de alimentos", "publicidade de alimentos”, "rótulos alimentares”, em português e em inglês.

Síntese dos dados: O governo brasileiro, nos últimos anos, tem promulgado ações de promoção de saúde que visam ao combate da obesidade infantil, como o Programa Saúde na Escola, o Programa Nacional de Alimentação Escolar, a Regulamentação dos Alimentos Comercializados nas Cantinas Escolares, o Projeto Escola Saudável, a Promoção da Alimentação Saudável nas Escolas, os Dez Passos para a Promoção da Alimentação Saudável nas Escolas e a Regulamentação de Propaganda e Publicidade de Alimentos. Observa-se a necessidade de implementar e de fiscalizar as leis e regulamentações para o controle da obesidade infantil no Brasil, além de promover a alimentação saudável, nos aspectos que envolvem o público infantil.

Conclusões: $\mathrm{O}$ projeto, o planejamento, a implementação e a gestão dessas políticas devem se apoiar na busca da transformação do problema social da obesidade.

Palavras-chave: obesidade; políticas públicas; criança; política de saúde; recomendações nutricionais.

\section{ABSTRACT}

Objective: To analyze the Brazilian nutrition public policies on the control of childhood obesity.

Data sources: Articles, essays, reviews and laws in SciELO, Lilacs, PubMed, Virtual Health Library, System Health Legislation and Sanitary Surveillance Legislation between 1990 and 2010, about Brazilian nutrition public policies for control of childhood obesity were searched. The keywords "obesity", “overweight”, “child”, "schoolchildren”, "public policy”, "health policy", "nutrition policy", "school canteen", "food school", "food advertising", "food labels", in Portuguese and English.

Data synthesis: The Brazilian government was active in designing health promotion programs aimed at combating childhood obesity in the recent years, such as the School Health Program, the National School Feeding Program, the Food Regulatory Marketed in School, the Healthy School Project, Promoting Healthy Eating in Schools, Ten Steps for Promoting Healthy Eating in Schools, and the Regulation of Advertising and Advertising of Foods. There is a need for implementation and enforcement of these laws and regulations for the control of childhood obesity in Brazil in order to promote healthy feeding habits.

Conclusions: The design, planning, implementation and management of these policies should aim the transformation of the social problem of obesity.

Key-words: obesity; public policies; child; health policy; nutrition policy.
Instituição: Universidade de Brasília (UnB) e Universidade Federal de Viçosa (UFV), Viçosa, MG, Brasil

'Doutorando em Ciências da Saúde pela UnB, Brasília, DF, Brasil

${ }^{2}$ Doutoranda em Nutrição Humana pela UnB; Nutricionista do Ministério da Saúde, Brasília, DF, Brasil

${ }^{3}$ Doutora em Ciências da Saúde pela Universidade Federal de Minas Gerais (UFMG); Professora do Departamento de Nutrição e Saúde da UFV, Viçosa, MG, Brasil

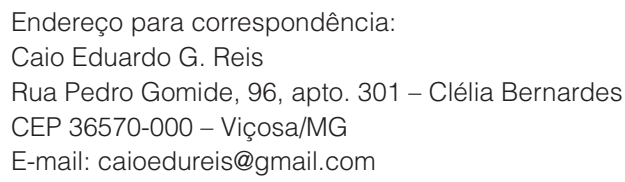

Endereço para correspondência:

Caio Eduardo G. Reis

Rua Pedro Gomide, 96, apto. 301 - Clélia Bernardes

CEP 36570-000 - Viçosa/MG

E-mail: caioedureis@gmail.com

Conflito de interesse: nada a declarar

Recebido em: 28/6/2010

Aprovado em: 12/1/2011 


\section{Introdução}

Por meio da lei n. ${ }^{\circ}$ 8.080/1990 é regulado o Sistema Único de Saúde (SUS) como estratégia para atenção e cuidado à saúde, integrando a seguridade social e baseando-se nos princípios da universalidade, equidade e integralidade, devendo atuar na formulação e no controle das políticas públicas de saúde ${ }^{(1)}$. Nesse contexto, surge a Política Nacional de Alimentação e Nutrição (PNAN, portaria n. $\left.{ }^{0} 710 / 1999\right)$, que formula os requisitos básicos para a promoção e a proteção à saúde, atestando o compromisso do Ministério da Saúde em controlar os males relacionados à alimentação e nutrição já configurados no Brasil ${ }^{(2)}$.

Os panoramas mundial ${ }^{(3,4)}$ e brasileiro ${ }^{(5,6)}$ da obesidade têm se revelado como um novo desafio para a saúde pública, uma vez que sua incidência e sua prevalência têm crescido de forma alarmante nos últimos 30 anos. A mudança do perfil nutricional que se desenha no Brasil revela a importância de um modelo de atenção à saúde que incorpore ações de promoção da saúde, prevenção e tratamento da obesidade e de doenças crônicas não transmissíveis ${ }^{(7)}$.

É importante ressaltar que o excesso de peso na infância predispõe a várias complicações de saúde, como: problemas respiratórios, diabetes melito, hipertensão arterial, dislipidemias, elevando o risco de mortalidade na vida adulta ${ }^{(8,9)}$. Segundo a Pesquisa de Orçamentos Familiares (POF), realizada em 2008-2009, a prevalência de excesso de peso (índice de massa corporal - IMC/idade $\geq 1$ escore $Z$ ) e obesidade (IMC/idade $\geq 2$ escores $Z$ ) em crianças de cinco a nove anos foi de $33,5 \%$ e $14,3 \%$, respectivamente. A obesidade correspondeu a cerca de um terço do total de casos de excesso de peso no sexo feminino e quase metade no masculino. A prevalência do excesso de peso oscilou de 25 a $30 \%$ nas Regiões Norte e Nordeste e de 32 a 40\% nas Regiões Sudeste, Sul e Centro-Oeste ${ }^{(10)}$. Em recente publicação, Reis et al mostraram que o excesso de peso tende a ser mais frequente no meio urbano do que no rural, em particular nas Regiões Norte, Nordeste e Centro-Oeste. Com magnitude menor, a prevalência da obesidade mostrou distribuição geográfica semelhante à observada para o excesso de peso ${ }^{(11)}$.

Nesse contexto, políticas públicas e programas de promoção da saúde, visando a hábitos alimentares saudáveis e práticas de atividades físicas regulares, são necessários para combater essa realidade. Medidas governamentais já foram tomadas a fim de controlar o aumento da prevalência da obesidade entre as crianças brasileiras. Nessa lógica, são importantes as políticas públicas que buscam atuar na prevenção e no controle da obesidade infantil. O objetivo do presente estudo foi analisar criticamente as políticas públicas de nutrição brasileiras para o controle da obesidade infantil.

\section{Método}

A pesquisa foi realizada com a busca de artigos, ensaios, resenhas, resoluções e legislações publicados entre janeiro de 1990 e dezembro de 2010 nas bases de dados eletrônicas Scientific Electronic Library Online Brasil (SciELO), Lilacs (Literatura Latino-Americana e do Caribe em Ciências da Saúde) e PubMed (US National Libary of Medicine, Bethesda, MD), Biblioteca Virtual em Saúde (BVS, Ministério da Saúde, Brasil), Sistema de Legislação em Saúde (Saude-legis, Ministério da Saúde, Brasil) e Legislação em Vigilância Sanitária (e-legis, Agência Nacional de Vigilância Sanitária - ANVISA, Brasil), que abordaram políticas públicas de nutrição brasileiras no controle da obesidade infantil. Foram utilizadas as palavras-chaves: "obesidade", "sobrepeso", "criança”, "escolar(es)", "políticas públicas”, "política de saúde”, "política de nutrição", "cantina escolar”, "alimentação escolar", "propaganda de alimentos", publicidade de alimentos", "rótulos alimentares" e seus respectivos termos em inglês. Por meio da leitura dos documentos encontrados, foram escolhidos os que continham informações de acordo com os propósitos da pesquisa.

\section{Resultados e Discussão}

Ações para o tratamento da obesidade infantil têm mostrado efeitos positivos em vários estudos ${ }^{(12)}$. As pesquisas de intervenção centram seus estudos em comunidades e no ambiente escolar, mostrando resultados concretos $^{(13)}$. De acordo com Sargent $e t$ al, a atenção primária representa uma boa oportunidade de intervenção contra a obesidade infantil, mas é preciso um modelo eficaz de atuação. É necessária uma equipe de profissionais de saúde treinada, opções para mudança de hábitos de vida, levando a alterações de comportamento por meio da combinação de aconselhamento, educação, com recursos audiovisuais, apoio e motivação ${ }^{(14)}$.

\section{Políticas Públicas de Nutrição}

Como em outros países em desenvolvimento, a transição nutricional no Brasil é marcada pela presença concomitante de desnutrição, obesidade e doenças carenciais específicas ligadas à má nutrição. Dada a complexidade do quadro epidemiológico atual e de seus determinantes, uma única 
medida não é suficiente para melhorar o perfil nutricional de nossa população. As intervenções devem integrar ações de incentivo (difundem informação e motivam os indivíduos para adoção de práticas saudáveis); apoio (visam facilitar opções saudáveis entre pessoas que já estejam motivadas) e proteção à saúde (objetivam evitar a exposição de indivíduos e coletividades a fatores que estimulem práticas não saudáveis) ${ }^{(15)}$.

As políticas públicas voltadas para a saúde têm sido de grande importância para a população do país, mesmo decente das dificuldades de sua plena implementação. Historicamente, as políticas públicas no Brasil vêm sendo realizadas por meio de práticas assistencialistas, refletindo-se em relações que não incorporam o reconhecimento do direito à saúde. A função social das políticas tem sido alterada no que diz respeito à qualidade, quantidade e variedade ${ }^{(16)}$.

As políticas públicas em saúde integram o campo de ação social do Estado, orientado para a melhoria das condições de saúde da população. Sua tarefa específica consiste em organizar as funções públicas governamentais para promoção, proteção e recuperação da saúde dos indivíduos e da coletividade. Conforme a Constituição Federal promulgada em 1988, essas políticas orientam-se pelos princípios da universalidade e equidade no acesso às ações e serviços, e pelas diretrizes de descentralização da gestão, de integralidade do atendimento e de participação da comunidade na organização de um sistema único de saúde no território nacional ${ }^{(17)}$.

A PNAN é uma política social inserida no gênero das políticas públicas. Baseia suas ações em sete diretrizes, sendo elas: 1) estímulo às ações intersetoriais com vistas ao acesso universal aos alimentos; 2) garantia de segurança e qualidade dos alimentos e da prestação de serviços nesse contexto; 3) monitoramento da situação alimentar e nutricional; 4) promoção de práticas alimentares e estilos de vida saudáveis; 5) prevenção e controle dos distúrbios nutricionais e doenças nutricionais; 6) promoção do desenvolvimento de linhas de investigação e desenvolvimento; 7) capacitação de recursos humanos em saúde e nutrição.

Tais diretrizes deixam claro o entendimento do governo federal sobre a coexistência da privação e do excesso alimentar da população, propondo como estratégia fundamental a promoção da alimentação saudável. Apesar de a PNAN se voltar ao conjunto da sociedade brasileira, os principais programas e ações são focalizados e seletivos. São eles: Programa Nacional de Controle da Deficiência da Vitamina A, o Programa de Controle da Anemia Ferropriva e o Sistema de Vigilância Alimentar e Nutricional ${ }^{(18)}$.
A PNAN, ao direcionar esforços para a construir de uma agenda integrada da nutrição, não deixa dúvidas quanto à gravidade do problema representado pela obesidade em nosso meio. A PNAN reconhece, também, a natureza complexa da obesidade e define um conjunto de ações, no âmbito da saúde e de outros setores, para assegurar ambientes propícios a padrões saudáveis de alimentação e nutrição para todos ${ }^{(19)}$. Passos importantes nessa direção foram dados recentemente, como a inclusão de metas nacionais para reduzir a obesidade no Plano Nacional de Saúde, a aprovação de diretrizes nacionais para a alimentação saudável, o repasse de recursos federais para o financiamento de ações específicas de promoção de alimentação saudável e de atividade física nos municípios e a resolução da ANVISA, que regulamenta a publicidade de alimentos não saudáveis. No âmbito intersetorial, destaca-se a adoção de políticas de segurança alimentar e nutricional, bem como a integração do Programa Nacional de Alimentação Escolar (PNAE) com a produção local de alimentos e a agricultura familiar, favorecendo a oferta de frutas e hortaliças nas escolas e comunidades ${ }^{(20)}$.

\section{Alimentação escolar}

A criação do Programa Saúde na Escola, instituído por decreto presidencial n. ${ }^{\circ}$ 6.286/2007(21), resultante do trabalho entre os Ministérios da Saúde e da Educação, objetiva a ampliação das ações específicas de saúde aos alunos da rede pública de ensino. Avaliação das condições de saúde, promoção e prevenção da saúde, educação permanente e capacitação dos profissionais, monitoramento e análise da saúde dos estudantes são ações propostas pelo programa. O PNAE $^{(22)}$ é gerenciado pelo Fundo Nacional de Desenvolvimento da Educação (FNDE) e visa à transferência de recursos financeiros aos Estados e municípios, destinados a suprir, parcialmente, as necessidades nutricionais dos alunos. A lei n. ${ }^{\circ} 11.947 / 2009^{(23)}$ dispõe sobre o atendimento da alimentação escolar e do Programa Dinheiro Direto na Escola aos alunos da educação básica. Essa lei determina a utilização de, no mínimo, 30\% dos recursos repassados pelo FNDE para compra de produtos da agricultura familiar e do empreendedor familiar rural para a alimentação escolar, beneficiando não só a alimentação dos alunos, mas também os produtores da agricultura familiar. Esse programa é considerado um dos maiores programas na área de alimentação escolar no mundo e é o único com atendimento universalizado. Alimentação comercializada em escolas deve conter qualidade nutricional adequada, pois a formação de hábitos alimentares errôneos pode comprometer a saúde na infância e na vida adulta. A 
merenda escolar é de extrema importância para atender os requisitos nutricionais das crianças em fase escolar, porém, muitas não consomem a merenda oferecida pela escola, mas sim lanche levado de casa ou vendido em cantinas escolares, desperdiçando, dessa forma, recursos públicos, além de contribuir para uma alimentação não saudável e consequentes complicações de saúde.

O PNAE objetiva garantir aos escolares o acesso a uma melhor alimentação de forma permanente e, assim, contribuir para o melhor desempenho escolar e para a redução da evasão. Pode-se considerar que o PNAE atua na promoção da segurança alimentar e nutricional para esse grupo social prioritário. Entretanto, o adequado planejamento dos cardápios a serem servidos aos escolares e o acompanhamento da execução dos mesmos são importantes para o alcance dos objetivos do programa, na medida em que há oferta nutricional mínima a ser garantida, além de ser considerado como um instrumento para formação de bons hábitos alimentares entre os beneficiários ${ }^{(24)}$.

Em avaliação do PNAE em 45 municípios do Estado da Bahia, entre 1995 e 2002, Santos et al verificaram que a cobertura do programa foi bastante significativa. A aceitabilidade da alimentação oferecida foi considerável, resultado esperado frente à situação de pobreza do conjunto dos municípios assistidos. Entretanto, a focalização deixa a desejar no interior da Bahia, onde se encontrou maior proporção de escolas com PNAE entre as frequentadas por alunos de maior poder aquisitivo. A irregularidade na oferta do benefício ainda enfrenta problemas importantes, comprometendo a aspiração do programa de ser efetivo como política social universal e um direito da criança, na perspectiva da promoção da segurança alimentar e nutricional. Além disso, problemas foram relatados quanto à insuficiência de infraestrutura, uma vez que a gestão descentralizada demanda investimentos em nível local para o provimento das instalações, equipamentos e do pessoal para o preparo e distribuição das refeições, sendo essa realidade mais complexa em áreas rurais ${ }^{(24)}$.

Em março de 2009, o Instituto Brasileiro de Geografia e Estatística (IBGE) iniciou a coleta de dados da Pesquisa Nacional de Saúde do Escolar (PeNSE), que avaliou 618.500 alunos de escolas públicas e privadas de todo país que frequentam o nono ano do ensino fundamental - a maioria na faixa de 13 a 15 anos. Foram avaliadas questões sobre alimentação, atividade física, condições de vida e violência, entre outras. Os resultados mostram que $57 \%$ dos estudantes são inativos ou insuficientemente ativos em termos de prática física, considerando somente as alunas, o percentual chega a
$69 \%$ e, dentre os meninos, $44 \%$. O consumo de guloseimas (balas, bombons, chicletes, doces, chocolates e pirulitos) e de refrigerantes superou o de frutas in natura. $\mathrm{O}$ consumo de frutas in natura foi de $32 \%$, enquanto o de guloseimas em cinco ou mais dias na semana foi de $51 \%$, refrigerantes $37 \%$, batata frita $5 \%$ e salgados fritos $13 \%{ }^{(25)}$.

\section{Cantinas escolares}

Os elevados índices de sobrepeso e obesidade em escolares no Brasil e no mundo suscitaram a necessidade de implementar estratégias de promoção da alimentação saudável para essa população. Santa Catarina foi o primeiro Estado brasileiro a criar uma legislação específica (lei estadual 12.061/2001) (26) para regulamentar os alimentos comercializados nas cantinas escolares. A fiscalização desses estabelecimentos é necessária, juntamente a ações de educação nutricional, visando promover hábitos alimentares mais saudáveis e fornecer subsídios para políticas de alimentação escolar ${ }^{(27)}$. Diversos Estados (Paraná, Mato Grosso, São Paulo, Rio Grande do Sul, entre outros) adotaram a ideia e vem regulamentando esse comércio dentro do ambiente escolar ${ }^{(28)}$. No Distrito Federal, o projeto de lei n. ${ }^{\circ} 3.695 / 2005$ dispõe sobre a promoção da alimentação saudável nas escolas de sua rede de ensino, indica que a cantina escolar deve ser administrada por pessoa capacitada em aspectos de alimentação e nutrição, relevantes para o exercício do comércio de alimentos destinados à população infanto-juvenil ${ }^{(29)}$.

Gabriel et al ${ }^{(30)}$, ao avaliarem o cumprimento da lei no Estado de Santa Catarina, observaram que a presença da cantina foi significativamente maior no setor privado $(p<0,001)$, comparada ao público. A maioria das cantinas $(68 \%)$ não vende salgadinhos fritos, refrigerantes, pipocas industrializadas, balas, pirulitos e gomas de mascar. Esses resultados mostram que, após oito anos da promulgação da lei, a fiscalização desses estabelecimentos é necessária, juntamente com ações educativas direcionadas aos proprietários das cantinas, alunos e administração escolar.

Nesse contexto, surgiu o Projeto Escola Saudável, que objetiva a implementação de alimentação saudável e atividades físicas regulares, com incentivo às hortas escolares, sendo a escola o agente de mudanças de hábitos alimentares e de vida. Ações vinculadas às universidades e aos órgãos não governamentais vêm auxiliando na melhoria da qualidade da alimentação oferecida pelas escolas pública. Um exemplo é o projeto de extensão da Universidade de Brasília (UnB): “A Escola Promovendo Hábitos Alimentares Saudáveis". Desde 2001, esse projeto promove, no Distrito Federal, a 
formação de bons hábitos alimentares na comunidade escolar, no contexto da promoção de estilos de vida saudáveis e prevenção de doenças crônicas não transmissíveis, com a capacitação de educadores do ensino infantil e fundamental até a quinta série e de donos de cantina escolar. A avaliação do projeto foi positiva, havendo ampliação significativa dos conhecimentos $(p<0,05)$ dos participantes após os três módulos desenvolvidos quanto à implantação da cantina saudável, além de resultados positivos observados ao se compararem os períodos anterior e posterior à capacitação, destacando o ambiente escolar como um espaço de promoção de alimentação saudável ${ }^{(31)}$.

Foi promulgada, em 2006, a Portaria Interministerial n. ${ }^{\circ}$ 1.010, que institui as diretrizes para a Promoção da Alimentação Saudável nas Escolas de educação infantil, fundamental e nível médio das redes públicas e privadas em todo o Brasil ${ }^{(32)}$. Suas diretrizes baseiam-se nas ações de educação alimentar e nutricional, estímulo à produção de hortas escolares, implantação de boas práticas de manipulação, monitoramento da situação nutricional e regulamentação do comércio de alimentos: restrição ao comércio no ambiente escolar de alimentos e preparações com altos teores de gordura saturada, gordura trans, açúcar livre e sal, com incentivo ao consumo de frutas e hortaliças.

Em todo o mundo, as escolas têm sido os alvos de propostas para combater a obesidade infantil, sendo fundamental que as avaliações futuras dessas políticas possam medir a eficácia em longo prazo das políticas de alimentação escolar no combate, tanto da inadequação da ingestão alimentar, quanto nos índices de sobrepeso e obesidade. Em recente revisão sistemática sobre as políticas de alimentação e nutrição no âmbito escolar, constatou-se a falta de resultados consistentes para garantir a eficácia das regulamentações ${ }^{(33)}$.

\section{Dez Passos para a Promoção da Alimentação Saudável nas Escolas}

Os Dez Passos para a Promoção da Alimentação Saudável nas Escolas ${ }^{(34)}$ foi elaborado com o objetivo de propiciar a adesão da comunidade escolar a hábitos alimentares saudáveis e promoção da saúde. Consistem num conjunto de estratégias que devem ser implementadas a fim de permitir a formulação de ações/atividades de acordo com a realidade de cada local. As estratégias precisam ser definidas em conjunto com a comunidade, favorecendo escolhas saudáveis, reforçando a promoção da saúde e a alimentação saudável no ambiente escolar. Tais estratégias devem também sensibilizar e capacitar os profissionais envolvidos com alimentação na escola para produzir e oferecer alimentos mais saudáveis, adequando os locais de produção de refeições às boas práticas de fabricação. Além disso, tais estratégias devem ainda restringir a oferta, a promoção comercial e a venda de alimentos ricos em gorduras, açúcares e sal, oferecendo opções de alimentos e refeições saudáveis, aumentando a oferta e a promoção do consumo de frutas e hortaliças, com ênfase nos alimentos regionais. Finalmente, esse conjunto visa desenvolver um programa contínuo de promoção de hábitos alimentares saudáveis, considerando o monitoramento do estado nutricional dos escolares, com ênfase em ações de diagnóstico, prevenção e controle dos distúrbios nutricionais.

\section{Propagandas de alimentos}

A propaganda de alimentos e sua influência nas escolhas alimentares têm sido alvo de discussões frequentes, sendo atribuída à propaganda parte da responsabilidade pelos problemas de má alimentação da população infantil. Tal situação tem levado a iniciativas governamentais que visam disciplinar as propagandas de alimentos, principalmente aquelas destinadas a esse público. Nos últimos anos, a publicidade e a propaganda de alimentos são focos de discussões internacionais, especialmente as voltadas para o público infantil, considerando o encorajamento ao consumo de alimentos ricos em gorduras, açúcar e sal, bem como a influência que os meios de comunicação exercem nas práticas alimentares infantis ${ }^{(35)}$.

Faz-se necessário um aprofundamento do diálogo do setor público com as indústrias de alimentos e dos meios de comunicação e marketing, com o objetivo de mudar progressivamente a demanda e eliminar a promoção de produtos alimentícios e de bebidas que contribuam para o aumento do peso e suas possíveis comorbidades. A regulamentação da publicidade de propagandas tem como base a promoção da saúde e a prevenção de doenças a partir da concretização do direito humano à alimentação adequada $\mathrm{e}$ à segurança alimentar e nutricional. Muitos países controlam e proíbem a publicidade de alimentos na televisão. Há outros que controlam essas propagandas em determinados horários, como o de programação infantil ${ }^{(36)}$.

Resultados apontaram a prevalência de comerciais de alimentos no período da manhã, voltados principalmente ao público infantil, sendo a maioria desses produtos ricos em açúcares e gorduras. Constatou-se que as mensagens são fundamentalmente afetivas, com ênfase no sabor dos alimentos e no prazer, havendo pouca referência aos aspectos nutricionais e de saúde. Grande parte dos comerciais apresenta 
elementos discutíveis quanto à questão ética. Considera-se que somente ações conjuntas, de ordem pública e privada, são capazes de auxiliar na promoção de hábitos alimentares mais saudáveis. Resultados parciais de uma pesquisa realizada pelo Observatório de Políticas de Segurança Alimentar e Nutrição da UnB alertou para o problema depois de avaliar mais de quatro mil horas de transmissão televisiva. Os pesquisadores identificaram que as propagandas mais frequentes são de alimentos com altos teores de gorduras, açúcares e sal (fast food, guloseimas, sorvetes, refrigerantes, sucos artificiais, salgadinhos de pacote, biscoitos doces e bolo). Somados, esses anúncios alcançam $72 \%$ do total de publicidade nos horários em que as crianças geralmente estão em casa: das $14 \mathrm{~h} 30$ às 18h30. Observa-se, portanto, um estímulo ao consumo de alimentos que contribuem para o aumento da prevalência de doenças como obesidade, hipertensão e diabetes melito. É preciso discutir sobre a urgência de se regulamentar a publicidade de alimentos ${ }^{(37-39)}$.

\section{Regulamentação da publicidade de alimentos}

A resolução 24/2010 da ANVISA ${ }^{(40)}$ dispõe sobre a oferta, a propaganda, a publicidade, a informação e outras práticas correlatas, cujo objetivo seja a divulgação e a promoção comercial de alimentos com quantidades consideradas elevadas de açúcar, gordura saturada, gordura trans, sódio e de bebidas com baixo teor nutricional, em quaisquer que sejam as formas e os meios de sua veiculação (rádio, televisão, cinema, jornais, revistas, demais impressos, folders, panfletos, filipetas, displays, internet, entre outros). O objetivo central do regulamento é impedir o aumento das doenças crônicas não transmissíveis, principalmente em crianças e adolescentes, público considerado de maior vulnerabilidade às mensagens publicitárias. Com isso, toda e qualquer propaganda desse tipo deveria vir acompanha de alertas sobre os perigos do consumo excessivo desses nutrientes, por meio de mensagens de acordo com as descritas na lei. Além disso, proíbe a utilização de figuras, desenhos, personalidades e personagens que sejam cativos ou admirados por esse público-alvo.

Outra ação governamental que está em elaboração é o projeto de lei 5.921/2001 ${ }^{(41)}$, que determina: "fica proibida qualquer tipo de publicidade, especialmente as veiculadas por rádio, televisão e internet, de produtos ou serviços dirigidos à criança, no horário compreendido entre sete e 21 horas”. É justamente nesse horário que as crianças mais vêem televisão e os programas infantis veiculam as propagandas para esse público. Outro projeto de lei ( $\left.n^{\circ} 150 / 2009\right)^{(42)}$ do Senado Federal regulamenta a propaganda de alimentos não saudáveis destinados às crianças, a fim de restringir a propaganda daqueles com alto teor de gordura trans e saturada, sódio e bebidas com baixo valor nutricional. Um dos itens do projeto defende a exibição da propaganda apenas entre 21 e seis horas e, mesmo assim, acompanhada de mensagens de advertência.

\section{Promoção da Saúde}

A promoção da saúde é entendida como um campo conceitual, político e metodológico para analisar e atuar sobre as condições sociais, críticas para melhorar a situação de saúde e a qualidade de vida das pessoas ${ }^{(43)}$.

A Estratégia Global em Alimentação, Atividade Física e Saúde, aprovada em 2004 pela Assembleia Mundial da Saúde, com o firme apoio do governo brasileiro, chama atenção para o aumento explosivo da obesidade e sobre o impacto desse aumento na incidência de várias doenças crônicas (diabetes melito, hipertensão, doenças cardiovasculares e certos tipos de câncer), na expectativa de vida da população e nos custos dos serviços de saúde. O enfrentamento do problema pelo governo nacional requer políticas públicas e ações intersetoriais, que vão além de informar e educar os indivíduos. Tais políticas devem, essencialmente, propiciar um ambiente que estimule, apoie e proteja padrões saudáveis de alimentação e atividade física. Por exemplo, por meio de medidas fiscais que tornem mais acessíveis os alimentos saudáveis, de normas que limitem a publicidade de alimentos não saudáveis e de intervenções no planejamento urbano que facilitem a prática cotidiana de atividade física ${ }^{(44)}$.

O Projeto Vida Saudável é um exemplo de política pública desenvolvida pela prefeitura de Curitiba (PR) a partir de 2000, que transforma espaços públicos, como parques e espaços comunitários, em áreas de democratização da educação em saúde e de estímulo à atividade física, adoção de hábitos alimentares saudáveis, atividades culturais e de lazer, educação ambiental, entre outros, criando oportunidades de participação que favorecem o "empoderamento" e a aquisição de habilidades da população para uma vida mais saudável. Contudo, não bastam a intenção e a visão bem-intencionadas, delegando à sociedade civil a responsabilidade pela manutenção de tais práticas saudáveis. É preciso firme investimento público, com uma ação consistente e estruturada na afirmação de responsabilidades do poder público em políticas intersetoriais articuladas ${ }^{(43)}$.

Criar espaços de troca e construção constante de saberes, linguagens e práticas, buscando o desenvolvimento pessoal e social, habilitando as populações para opções por uma vida mais saudável, significa fazer com que, em cada um dos 
microssistemas (escola, casa, organizações comunitárias e instituições), sejam organizadas ações de Promoção da Saúde. O impacto de uma intervenção de promoção à saúde em uma perspectiva ampla certamente poderá refletir nos gastos do SUS em relação às enfermidades e mortes evitáveis, na melhoria da qualidade de vida da população e na compreensão de que manter a saúde é uma tarefa que exige um esforço em conjunto, mobilizando o indivíduo, a comunidade, o governo em torno de ideias e ideais ${ }^{(45)}$.

A regularidade de um comportamento e a transformação do mesmo em hábito implica múltiplas questões. Motivação para a ação, percepção dos benefícios, adequação às necessidades pessoais e satisfação com a atividade executada são itens fundamentais para que isso possa acontecer ${ }^{(45)}$. A questão-chave continua sendo a capacidade de motivar suficientemente todos os atores envolvidos, fornecendo clareza conceitual, recursos de conhecimento e transparência de informações ${ }^{(43)}$.

O novo paradigma da saúde é: como organizar medidas capazes de promover a saúde e prevenir doenças, a fim de garantir modos mais saudáveis de viver e também diminuir gastos relativos ao setor saúde, com baixas taxas de morbimortalidade por doenças crônicas não transmissíveis? Como se adequar às mudanças necessárias para a produção de alimentos menos processados, acessíveis do ponto de vista financeiro e com teores reduzidos de sal, açúcar e gorduras em geral, sem abrir mão do lucro esperado ${ }^{(46)}$ ?

Faz-se necessária uma reflexão sobre aspectos da segurança alimentar e nutricional, ainda pouco trabalhados na produção científica nacional. A alimentação fora de casa é um deles, que representa uma importante faceta do comportamento alimentar atual e, somente na versão 2008-2009 da POF/IBGE, esses dados foram inclusos. Aspectos como a comida de rua, a alimentação em restaurantes comerciais e coletivos, os patrimônios gastronômicos regionais, dentre outros, são comumente alvo somente de pesquisas de qualidade sanitária, reduzindo o foco da contribuição para a segurança alimentar e nutricional ${ }^{(47)}$.

A estratégia de promoção da alimentação saudável é a que melhor condiz com o complexo perfil nutricional de nossa população. Alguns exemplos de medidas de promoção da alimentação saudável dirigidas ao ambiente: 1) taxação e consequente aumento de preço dos produtos ricos em gorduras, açúcares e sal; 2) revisão dos subsídios à produção de alimentos, valorizando-se a produção de frutas e hortaliças, e produtos ecologicamente sustentáveis; 3) regulamentação da publicidade de alimentos; 4) regulamentação de venda e propaganda de alimentos no ambiente escolar; 5) implementação de medidas que facilitem o acesso físico a frutas e hortaliças em programas públicos de alimentação (como o PNAE) em áreas urbanas e rurais com pouco ou nenhum acesso a esses alimentos.

Alguns exemplos de medidas dirigidas aos indivíduos são: 1) ampliação do nível de conhecimento da população sobre alimentação; 2) valorização da cultura alimentar; 3) promoção da cidadania; 4) desenvolvimento de habilidades que ampliem a autonomia nas escolhas alimentares e no preparo de refeições saudáveis.

A ação em parceria contribui para a consolidação de medidas consistentes, inovadoras e condizentes com o complexo perfil epidemiológico de nossa população ${ }^{(15)}$.

\section{Conclusões}

Orientações nutricionais, adequação da merenda escolar e número suficiente de nutricionistas são atitudes que precisam ser reconsideradas pela rede escolar pública. Os resultados apontam a necessidade das atividades de educação e monitoramento nutricional e da atuação efetiva da escola junto à secretaria de educação na vigilância epidemiológica e na implementação de intervenções eficazes. É responsabilidade do Estado promover o direito humano à alimentação adequada, incorporando às políticas públicas de nutrição o diálogo intersetorial para sua plena implementação. As políticas públicas são fundamentais para a garantia do direito humano à alimentação adequada, portanto, o projeto, o planejamento, a implementação e a gestão dessas políticas devem se apoiar na busca da transformação do problema social da desnutrição e obesidade.

Os elementos demonstrados no presente estudo analisaram as políticas públicas de nutrição, sinalizando para a necessidade da ampla implementação e regulamentação das leis para o controle da obesidade infantil no Brasil e promoção da alimentação saudável nos aspectos que envolvem o público infantil. O desafio é desenvolver, fomentar e articular ações eficientes para controlar o processo de transição nutricional, em um cenário de extrema desigualdade social. Diante dessa gama de elementos que permeia toda a estrutura das políticas públicas de saúde em nosso país e, especificamente, as de nutrição, espera-se que, em um futuro próximo, possa haver melhora significativa e que as classes populares tenham acesso a esse bem tão precioso para o bem-estar de toda coletividade. 


\section{Referências bibliográficas}

1. Brasil. Lei n. 8.080, de 19 de setembro de 1990. Dispõe sobre as condições para a promoção, proteção e recuperação da saúde, a organização e o funcionamento dos serviços correspondentes e dá outras providências. Brasília, DF: Diário Oficial da União, 1999.

2. Brasil - Ministério da Saúde. Portaria n. 710, de 10 de junho de 1999. Aprova a Política Nacional de Alimentação e Nutrição. Brasília (DF): Diário Oficial da União, 1999

3. Dietz WH. The obesity epidemic in young children. Reduce television viewing and promote playing. BMJ 2001;322:313-4.

4. Ebbeling CB, Pawlak DB, Ludwig DS. Childhood obesity: public-health crisis, common sense cure. Lancet 2002;360:473-82.

5. Brasil - Ministério do Planejamento, Orçamento e Gestão. Instituto Brasileiro de Geografia e Estatística. Estudo Nacional da Despesa Familiar - ENDEF: dados preliminares - consumo alimentar - antropometria. Rio de Janeiro: IBGE, 1977.

6. Brasil - Ministério do Planejamento, Orçamento e Gestão. Instituto Brasileiro de Geografia e Estatística. Pesquisa de Orçamentos Familiares - 2002-2003: antropometria e análise do estado nutricional de crianças e adolescentes no Brasil. Rio de Janeiro: IBGE, 2006.

7. Brasil - Ministério da Saúde. Secretaria de Atenção à Saúde/Departamento de Atenção Básica. Caderno de atenção básica - Obesidade. Brasília (DF): Ministério da Saúde, 2006.

8. Taddei JA. Epidemiologia da obesidade na infância. Pediatr Mod 1993;29:111-5

9. Adami F, Vasconcelos FA. Childhood and adolescent obesity and adult mortality: a systematic review of cohort studies. Cad Saude Publica 2008;24 (Suppl 4):s558-68.

10. Brasil - Ministério do Planejamento, Orçamento e Gestão. Instituto Brasileiro de Geografia e Estatística. Pesquisa de orçamentos familiares 2008-2009: Antropometria, estado nutricional de crianças, adolescentes e adultos no Brasil. Brasília (DF): IBGE, 2010.

11. Reis CE, Vasconcelos IA, Oliveira OM. Panorama do estado antropométrico dos escolares brasileiros. Rev Paul Pediatr 2011;29:108-16.

12. Oude Luttikhuis $H$, Baur L, Jansen H, Shrewsbury VA, O'Malley C, Stolk $\mathrm{RP}$ et al. Interventions for treating obesity in children. Cochrane Database Syst Rev 2009:CD001872.

13. Flynn MA, McNeil DA, Maloff B, Mutasingwa D, Wu M, Ford C et al. Reducing obesity and related chronic disease risk in children and youth: a synthesis of evidence with 'best practice' recommendations. Obes Rev 2006; 7 (Suppl 1):7-66.

14. Sargent GM, Pilotto LS, Baur LA. Components of primary care interventions to treat childhood overweight and obesity: a systematic review of effect. Obes Rev 2011;12:e219-e235.

15. Leão MM, Castro IR. Políticas públicas de alimentação e nutrição. In: Kac G, Sichieri R, Gigante DP. Epidemiologia nutricional. Rio de Janeiro: Fiocruz/ Atheneu; 2007. p. 519-41.

16. Traverso-Yepez MA. Dilemas na promoção da saúde no Brasil: reflexões em torno da política nacional. Interface 2007;11:223-8.

17. Oliveira DC, Sá CP, Gomes AM, Ramos RS, Pereira NA, Santos WC. Brazilian National Health Policy: health professionals' social representations. Cad Saude Publica 2008;24:197-206.

18. Brasil - Ministério da Saúde. Política Nacional de Alimentação e Nutrição. Brasília (DF): Ministério da Saúde; 1999.

19. Brasil - Ministério da Saúde. Política nacional de alimentação e nutrição. $2^{\mathrm{a}}$ ed. Série B. Textos Básicos de Saúde. Brasília (DF): Ministério da Saúde; 2003.

20. Viana AL, Fausto MC, Lima LD. Política de saúde e eqüidade. São Paulo em Perspectiva 2003;17:58-68.

21. Brasil. Decreto presidencial no. 6.286 de 05 de dezembro de 2007. Institui o Programa Saúde na Escola - PSE, e dá outras providências. Brasília (DF): Diário Oficial da União, 2007.

22. Brasil. Resolução/FNDE/CD/ no. 38 de 16 de julho de 2009. Dispõe sobre o atendimento da alimentação escolar aos alunos da educação básica no
Programa Nacional de Alimentação Escolar - PNAE. Brasília (DF): Diário Oficial da União, 2009.

23. Brasil. Lei no. 11.947, de 16 de junho de 2009. Dispõe sobre o atendimento da alimentação escolar e do Programa Dinheiro Direto na Escola aos alunos da educação básica. Brasília (DF): Diário Oficial da União, 2009.

24. Santos LM, Santos SM, Santana LA, Henrique FC, Mazza RP, Santos LA et al. Evaluation of food security and anti-hunger public policies in Brazil, 1995-2002. 4 - National School Nutrition Program. Cad Saude Publica 2007; 23:2681-93.

25. Brasil - Instituto Brasileiro de Geografia e Estatística [homepage on the Internet]. Pesquisa Nacional de Saúde do Escolar, 2009 [cited 2010 Mar 7] Available from: http://www.ibge.gov.br/home/presidencia/noticias/noticia visualiza.php?id_noticia $=1525$

26. Brasil. Lei no. 12.061, de 18 de dezembro de 2001. Dispõe sobre critérios de concessão de serviços de lanches e bebidas nas unidades educacionais localizadas no Estado de Santa Catarina. Brasília (DF): Diário Oficial do Estado de Santa Catarina, 2001.

27. Reis D, Matos CH, Henn R. Fulfilment of Law 12.061/2001 (Law of school snack bars) in public schools of Santa Catarina coast city. Nutrição em Pauta 2007;15:43-5

28. Brasil - Ministério da Saúde. Experiências estaduais e municipais de regulamentação da comercialização de alimentos em escolas no Brasil: identificação e sistematização do processo de construção e dispositivos legais adotados. Brasília (DF): Ministério da Saúde, 2007.

29. Brasil. Lei no. 3.695, de 8 de novembro de 2005. Dispõe sobre a promoção da alimentação saudável nas escolas da rede de ensino do Distrito Federal. Brasília (DF): Diário Oficial do Distrito Federal, 2005.

30. Gabriel CG, Vasconcelos FA, Andrade DF, Schmitz BA. First law regulating school canteens in Brazil: evaluation after seven years of implementation. Arch Latinoam Nutr 2009;59:128-38.

31. Schmitz BA, Recine E, Cardoso GT, Silva JR, Amorim NF, Bernardon R et al. Promotion of healthy eating habits by schools: a methodological proposal for training courses for educators and school cafeteria owners. Cad Saude Publica 2008;24 (Suppl 2):s312-22.

32. Brasil. Portaria Interministerial n. 1010 de 8 de maio de 2006. Institui as diretrizes para a Promoção da Alimentação Saudável nas Escolas de educação infantil, fundamental e nível médio das redes públicas e privadas, em âmbito nacional. Brasília (DF): Diário Oficial da União, 2006.

33. Jaime PC, Lock K. Do school based food and nutrition policies improve diet and reduce obesity? Prev Med 2009;48:45-53.

34. Brasil - Ministério da Saúde. Dez passos para a promoção da alimentação saudável nas escolas. Brasília: Ministério da saúde, 2004 Available from http://189.28.128.100/nutricao/docs/geral/dez_passos_pas_escolas.pdf

35. Hawkes C. Marketing food to children: changes in the global regulatory environment 2004-2006. Geneva: WHO; 2007.

36. Monteiro CA, Castro IR. Por que é necessário regulamentar a publicidade de alimentos. Cienc Cult 2009;61:56-9.

37. Observatório de Políticas de Segurança Alimentar e Nutrição, Universidade de Brasília [homepage on the Internet]. Monitoramento de propaganda de alimentos visando à prática da alimentação saudável [cited 2010 Mar 11] Available from: http://www.unb.br/fs/opsan/propaganda.pdf

38. Pontes TE, Costa TF, Marum AB, Brasil AL, Taddei JA. Nutritional guidance for children and adolescents and the new consumption patterns: advertising, packaging and labeling. Rev Paul Pediatr 2009;27:99-105.

39. Almeida SS, Nascimento PC, Quaioti TC. Amount and quality of food advertisement on Brazilian television. Rev Saude Publica 2002;36:353-5.

40. Brasil - Agência Nacional de Vigilância Sanitária. Resolução - RDC n² 24, de 15 de junho de 2010. Brasília (DF): Diário Oficial da União, 2010.

41. Brasil. Projeto de lei n. 5.921 de 2001. Proíbe a publicidade dirigida à criança e regulamenta a publicidade dirigida a adolescentes. Brasília (DF): Diário Oficial da União, 2001.

42. Brasil. Projeto de lei n. 150 de 2009. Introduz modificações na Lei no. 10.895, de 26 de dezembro de 1996, que instituiu o Fundo de Fomento Automotivo do 
Estado do Rio Grande do Sul - FOMENTAR/RS. Rio Grande do Sul: Diário Oficial da Assembléia Legislativa do Estado do Rio Grande do Sul, 2009.

43. Moysés SJ, Moysés ST, Krempel MC. Assessing the building process of health promotion public policies: the experience of Curitiba. Cienc Saude Coletiva 2004;9:627-41.

44. World health organization [homepage on the Internet]. Global strategy on diet, physical activity and health. Resolution of the World Health Assembly. Fifty-seventh World Health Assembly [cited 2010 Jul 20]. WHA57. Geneva, 2004. Available from: http://apps.who.int/gb/ebwha/pdf_files/WHA57/A57_R17-en.pdf
45. Moretti AC, Almeida V, Westphal MF, Bógus CM. Corporal practices/physical activity and public policies of health promotion. Saude Soc 2009;18:346-54.

46. Pinheiro AR, Carvalho DB. Estado e mercado: adversários ou aliados no processo de implementação da Política Nacional de Alimentação e Nutrição? Elementos para um debate sobre medidas de regulamentação. Saude Soc 2008;17:170-83.

47. Proença RP. From research on food security and nutrition in Brazil to the challenge of creating committees on feeding and nutrition. Cienc Saude Coletiva 2010;15:24-6. 